quite surprised that a tender place of which they were unaware should be made manifest to their feelings. These nerves normally are not tender on pressure, unless it be severe.

The shoulder-tip pain and the nerve-tenderness in these diseases are owing, as I believe they are in liver-diseases, ${ }^{*}$ to an extension of irritation or inflammatory action from the diseased organ along its pneumogastric twigs to the trunk of the par vagum. In a case in which the morbid state of the spleen has been intense and continued for a time, this irritation or inflammation passes up the trunk of the nerve to the base of the skull, and even to the origin of the par vagum and the two divisions of the spinal accessory, giving rise there to pains and other disturbances.

There can be little wonder that the external division of the latter nerve becomes affected, when we recollect the intimate incorporation of the internal division with the pneumogastric, and the connections of the internal and external divisions with each other. When the irritation of the pneumogastric has reached the connection of this nerve with the external division of the spinal accessory, it appears to be "shunted", or diverted in part along that division, which it causes to be tender along its whole course to the shoulder-tip, and is at times manifested as pain at that part.

This shoulder-pain in splenic diseases will limit, on the left side most commonly, as it does generally in hepatic diseases on the right side, the respiratory movements of the sterno-cleido-mastoid and trapezius muscles, and thus the splenic side of the chest will be kept more quiet than it would otherwise be. When liver and spleen are both diseased, then both sides may be similarly affected in their movements.

The above morbid state of the pneumogastric trunk may and does, in its upward course, affect various branches, as those of the stomach, liver, heart, lungs, and even ear, giving origin to symptoms characteristic of disorder of one or other, or of more than one, of these organs ; and an attentive observer may remark that many of the symptoms in cases of spleen-disease are attributable to parts which receive branches from the pneumogastric nerve. In fact, one has only to bear in mind the distribution of the offsets from that nerve to be able to say where pains and other morbid phenomena will in one case or another make their appearance.

There can be no doubt, however, that the morbid influence of a diseased spleen is felt and given notice of, not only by the pneumogastric, but by the sympathetic and spinal twigs of the organ, and through these several of the associated morbid symptoms are produced, especially those that arise in the spine and walls of the body, and in part also those that belong to the viscera themselves.

It is certainly worthy of notice how unilateral were many of these sympathetic affections in the majority of the cases given. It was most commonly the left side of the dorsal region of the spine, the left side of the chest, the left side of the neck, the left-shoulder-tip, scapula and arm, the left ear, the left side of the head, in front or behind the ear-even the left loin and leg, at times-that were the seats of suffering. Does it not seem very probable that in these cases the spleen derived its nerve-supply from the left vagus? The morbid influence, be it irritation or inflammation, which in these and similar cases constitutes the essence of sympathy of one diseased organ with another, appears to be conveyed from organ to organ along the nerves of the same side of the body more easily and naturally than from half organ to half organ along the nerves that cross the median line of the body.

There appears to be little doubt that, like the columns of the spinal cord, as they pass downwards through the medulla oblongata from the brain, the fibres of the par vagum and of the sympathetic, as they pass to the viscera, in part decussate on the median line, some from the right passing to the left side, and others from the left going to the right side, whilst the majority hold on a straight course on their own side. But we do not find anywhere described transverse commissural fibres passing direct from half organ to half organ across the median line of the body-such, for instance, as exist in the anterior and posterior parts of the optic chiasma, connecting together both sides of the optic parts of the middle brain behind and the two retinæ in front. Now, between the eyes there appears to be a greater sympathy in disorder than between the halves of the other divided organs, perhaps on account of the direct nervous connection between the retinæ; and it is perhaps on account of the want of such direct commissural fibres that sympathetic disturbance extends more easily along the nerves of one side instead of crossing the median line. * See a paper on Shoulder-tip Pain in Liver-Diseases (Brit. MEd. Journal,
October 22nd, 1870).

DR. WHEELER, R.N., formerly attached to the British Legation at Yedo, has been presented with a testimonial and a flattering address by the English residents on his removal to Yokohama.

\section{RETRO-PHARYNGEAL ABSCESS.*}

By MARTIN G. B. OXLEY, L.K.Q.C.P., Physician to the Liverpool Infirmary for Children.

RETRO-Pharyngeal abscess has been frequently and fully described, but cases of this disease are comparatively so rare and difficult to diagnose, that I think your time will not be wasted by my relating a case which has recently come under my notice, and also making a few remarks as to its nature and treatment. Dr. West, who mentions four cases which came under his observation-two being idiopathic, and the other two secondary to disease of the cervical vertebre- has been able to find records of only twenty-six cases of this disease in children under ten years. Dr. Abercrombie, in the Edinburgh Medical Fournal for 1819, under the head of cases of severe dyspnoea in children connected with a singular affection of the throat, relates three well marked cases of this disease. Dr. Fleming also, in the Dublin Medical Fournal for I840, under the heading-- "Practical Observations on Peculiar Affections of the Throat, arising from Abscess between the Pharynx and Spine, and occurring in Children and Adults, exemplified by Cases", gives two examples of this complaint. Since then, attention has been drawn from time to time to this troublesome form of abscess by various writers.

The case which I am about to relate is as follows. E. M., aged two years, was admitted into the Liverpool Infirmary for Children on March 3 rd, I874, suffering from great difficulty of breathing. The mother stated that the child had scarlet fever five months before, and since then had not been doing well. Two weeks before admission, she noticed that the child held her head rather stiffly, and breathed badly at night. This difficulty of breathing gradually increased; and the night before she was brought to the Infirmary, the mother "thought she would choke every moment".

On admission, I found a strumous-looking child, with wasted limbs and enlarged cervical glands, labouring in her breathing, and making a croupy sound at each inspiration, but not seeming in very much distress, able to swallow and cry. I considered it at first sight to be a case of enlarged tonsils, but was unable to get a very good view of the throat, on account of the large quantity of tenacious mucus which filled the larynx. I ordered the throat to be swabbed out with a strong solution of nitrate of silver. The next day, there was slight improvement ; and, on examination of the throat, nothing could be seen to account for the dyspnuea. The same treatment was continued for some days, the child in the meantime being neither better nor worse, and taking focd comfortably. On the I 4 th, eleven days after admission, the general condition remaining the same, I examined the throat with my finger, and found, at the back of the pharynx, on the right side, a firm elastic swelling, nearly filling up the throat. This swelling I punctured with a bistoury, and evacuated a large quantity of healthy pus. This was followed by immediate improvement in the breathing. The child continued better for two days, when the breathing again became laborious; and, on examination with the finger, for it was impossible to see anything, I found that the abscess had refilled. I again punctured it, and each day afterwards, for a short time, I took the precaution of pressing on the wall of the pharynx from below upwards, so as to empty the abscess. This latter is a very necessary precaution, as the abscess is very apt to refill. Since that time, she has had pus evacuated from the enlarged glands on each side of the neck. I had to cut very deeply to reach matter which was perfectly healthy, containing no cheesy substance. She had shortly after this an attack of scarlet fever followed by dropsy, from which she has made a fair recovery. I suppose the attack called by the mother scarlet fever must have been measles.

Retro-pharyngeal abscess may arise idiopathically, or from caries of the cervical vertebræ, or as the sequela of acute exanthems. When it arises from disease of the spine, which is very rare, it is slower in its course, and there is often paralysis of the upper extremities. When it is idiopathic, or a sequela of scarlet fever or measles, it forms more rapidly, and gives rise to greater difficulty in swallowing and breathing. The diagnosis is extremely difficult unless one is on the look out for it, as ocular examination gives us little or no assistance in a young child. We must suspect it if a child have difficult breathing or dysphagia, and a croupy cough, and if it hold its head stiffy. We must feel with the finger, when, if we find an elastic swelling filling up the pharynx, we may be certain of the nature of the disease. The difficulty of breathing is always aggravated on lying down. The disease differs from croup in this particular, as also in its lasting longer without destroying life; the child is also able to cry, the voice not being as a rule interfered with;

- Read before the Lancashire and Cheshire Branch. 
inspiration is exceedingly difficult, while expiration is comparatively easy.

The treatment is simple, and ought to be prompt; viz., puncture with a bistoury guarded to near the tip with adhesive plaster. This should be followed up each day by firm pressure with the finger, in order to prevent the refilling of the abscess.

\section{SELECTIONS FROM JOURNALS.}

\section{THERAPEUTICS.}

Ergotix and its Prfiparations. - At a meeting of the Berlin Medical Society (Allgemiene Medicin. Centralzeitung, Nos. 35 and 36), Dr. Wernich made a communication on the preparations of ergotin. Those which are in use have certain disadvantages when used for subcutaneous injection: they readily cause abscesses, produce much pain, and the solutions are not pure and are preserved with difficulty. Dr. Wernich has endeavoured to obtain a clear soluble preparation, perfectly capable of absorption, and possessing none of the above mentioned disadvantages. The part of the action of ergotin on the system which first comes under observation in its therapeutic use, is its influence on the blood-pressure in the vessels, of which the action on the uterus is a secondary result. A test of the therapeutic applicability of a preparation of ergotin is the production of definite visible changes in the vessels; and, as objects for this purpose, Dr. Wernich recommends the mesentery, the lung, and the web of the foot, of a curarised frog. $\mathrm{He}$ uses a watery extract prepared from powdered ergot, which has been first deprived of all fatty matter by ether, and then treated with alcohol. This watery extract, though efficacious, contains impurities, from which it may be cleansed by diffusion through parchment-paper. It then forms a perfectly clear solution in water, of a brown colour. The preparation made in this way is efficacious, and, in consequence of its purity, is preferable for subcutaneous injection: it keeps well, and does not produce pain.

Dry Cupping in Puerperal Convulsions, Etc.-Dr. Washington (Nashville Fournal, October 1873) details a case in which he immediately stopped puerperal convulsions by the vigorous use of dry cups, applied near the spine. Further, he says he has often used the same agent to bring on uterine pains in tedious labours; to cut short the sympathetic disturbances of pregnancy; to cure neuralgia, sick headache, total paralysis of the arm from gunshot-wounds, and cases of tetter of twenty and thirty years' duration.

\section{OBSTETRICS}

The Normal Temperature, Pulse and Respiration of Puerperal Women.-Dr. G. W. Linn (Philadelphia Medical Times, May 9th, 1874) has made careful observation, in Philadelphia Hospital, of twenty-five cases which were in every respect normal. He gives the following conclusions: I. The normal temperature of the puerperal woman is only about $0.6 \mathrm{deg}$. Fahr. higher than that of the healthy human being, if we accept the statement of Wunderlich that the mean normal temperature is $98.6 \mathrm{deg}$. Fahr.; 2. The normal pulse of the puerperal woman is not more frequent than that found under ordinary conditions in a state of perfect health; 3 . The number of respirations is increased, if the statement of physiologists be received that the number of respirations of the healthy woman ranges from eighteen to twenty per minute; 4 . The generally received opinion that the secretion of milk is attended by an increase of temperature of one or more degrees and an increase in the frequency of the pulse of ten or twelve beats per minute, is erroneous ; 5. A temperature of roo deg. Fahr. or a pulse of one hundred per minute in the lying-in woman is indicative of some pathological process which it behoves the accoucheur to discover at once, in order that proper measures may be taken to arrest its development and remove the evil.

Herpes Gestationis. - The peculiar features of this remarkable disease are summed up by Dr. Bulkley, in the American Fournal of Obstetrics, February 1874, as follows. I. It is an affection of the skin directly dependent upon the gravid state of the uterus, which may make its appearance at any period of gestation up to the seventh month. It generally continues until the organ is emptied of its contents, and has in a measure resumed its former state. It is very apt to recur at each successive conception. 2. The cutaneous manifestations are chiefly an intense irritation consisting of burning, itching, or stinging, and sometimes pains, with the development of erythema, papules, vesicles, and bullx up to the size of a hen's egg. These vesicles are commonly in groups, do not follow any definite nerve-tracks, appear first on the ex- tremities, and afterwards involve the larger part of the body. $\mathrm{Ex}$ haustion may ensue from cutaneous irritation, but the disease is nonfebrile. 3. The disease does not terminate at once after delivery, but slowly retrogrades. It is rare that any manifestation of the disease remains longer than a month after parturition. 4. It is sometimes accompanied or followed by other neurotic manifestations, as erythema, urticaria, and neuralgia. 5. This eruption has occasionally been the first indication that impregnation has taken place. 6 . The majority of cases have been uninfluenced by treatment, relief occurring only on the emptying of the uterus. 7. The children are not as a rule affected by the eruption in the mother. The indications for treatment are for soothing antipruritic local applications and powerful tonics, especially those directed to the nervous system.

\section{MEDICINE.}

Inflammation of Joints after DiarrhoeA. - During the abatement of an epidemic of diarrhœe which prevailed in the district of Minden in the latter part of the summer and in the autumn of 1872, Rapmund (Deutsche Klinik, No. 17, 1874) observed six convalescents, who, after exposure to cold or exertion, had acute articular inflammation. It always began in one knee or in both, and was either limited to this joint or attacked also the ankle; in one woman only, who had suffered from acute articular rheumatism eight years previously, both wrists were affected. In all the cases, improvement soon followed the use of appropriate treatment. There were no perspirations or complications, such as occur in acute articular rheumatism. These six cases were the only ones in which the affection of the joints was noticed in about four hundred cases of diarrhœa. Rapmund refers to other observations of the kind made by Braun (1833 and 1834 ) and Kräuter (I87I), but overlooks those related by Hütte (Centralblatt, I 869).

Apoplectiform Deafness.-Dr. S. M. Burnett (Nashville Medical and Surgical Fournal, May 1874), from an interesting discussion of Menière's disease, deduces the following conclusions. I. There is a series of symptoms comprising cerebral disturbance, nausea and vomiting, vertigo, loss of power of controlling equilibrium, deranged movement, tinnitus aurium, and impairment or destruction of hearing power. All these in their totality constitute the disease. 2. From the small number of post mortem examinations, we have no accurate knowledge of the exact pathological lesions. The facts already observed warrant us in considering it an extravasation, either hæmorrhagic or serous; and in the large majority of cases it is more or less sudden. The name apoplectiform deafness is accepted as most expressive of the accepted pathology. 3. The lesions are seated primarily in the auditory nervous apparatus. 4. In some cases, we can locate the lesions in the cochlea. 5. Lesion of the semicircular canals is followed by vertigo, deranged muscular action, and inability to control bodily equilibrium. Facts seem to show that these manifestations are reflex in character.

Relations of the Abdominal Branches of the PneumoGaSTRICS TO THE TREATMENT OF GASTRITIS, DIARRHCA, ETC. Dr. H. C. Hand, in the Northwestern Medical and Surgical Fournal, May I 874 , presents some considerations on this topic. He shows by quotation and experiment that the vaso-motor nerves are to be reached only through the sympathetic system; that their function is to reduce the size of the blood-vessels; that their stimulation diminishes the heat, colour, and functional activity of a part; that their paralysis by section dilates the capillaries, produces in the liver diabetes, and in the intestines a copious watery secretion. On the other hand, section of the pneumogastrics produces a directly opposite effect on the liver, stomach, and intestines, from section of the sympathetic-viz., contraction of the vessels, paleness of the mucous membrane, and diminution or abolishment of secretion, while their stimulation is followed by at least some of the phenomena produced by section of the sympathetic, $e_{.} g$., diabetes. In cases of prolonged and severe vomiting, he applied pressure on one of the vagi. The vomiting stopped, and the patients grew better or were entirely relieved. He suggests that pres sure upon the vagi would probably relieve cases of serous diarrhœa. Since the right vagus is distributed mostly to the small intestines, and the left to the stomach, it would seem probable that pressure upon the right would be most effective in diarrhœa, and upon the left for vomiting, etc., in gastritis. He suggests that a cone of ice might be substituted for pressure, if the latter were not appropriate. This pressure is most conveniently applied in the neck just below the angle of the jaw, the pulsation of the carotid supplying the best guide to the course of the nerve. 